\title{
Cannabinoid signaling promotes the reprogramming of Muller glia into
} proliferating progenitor cells.

6 Warren A. Campbell ${ }^{1}$, Sydney Blum ${ }^{1}$, Alana Reske $^{1}$, Thanh Hoang $^{2}$, Seth Blackshaw ${ }^{2}$,

91 Department of Neuroscience, College of Medicine, The Ohio State University,

10 Columbus, $\mathrm{OH}$

112 Solomon H. Snyder Department of Neuroscience, Johns Hopkins University School of

12 Medicine, Baltimore, MD University, College of Medicine, 3020 Graves Hall, 333 W. $10^{\text {th }}$ Ave, Columbus, OH 43210-1239, USA. Telephone: (614) 292-3524; Fax: (614) 688-8742; email: Andrew.Fischer@osumc.edu

Author Contributions: WAC and executed experiments, gathered data, constructed figures, and contributed to writing the manuscript. SB and AR executed experiments, and gathered data. AJF designed experiments, analyzed data, constructed figures, and wrote the manuscript.

Acknowledgements: This work was supported by RO1 EY022030-08, RO1 EY032141- 


\section{Abstract}

Endocannabinoids (eCB) are lipid-based neurotransmitters that are known to influence synaptic function in the visual system. eCBs are also known to suppress neuroinflammation in different pathological states. However, nothing is known about the roles of the eCB system during reprogramming of Müller glia (MG) into proliferating progenitor-like cells in the retina. Accordingly, we used the chick and mouse model to characterize expression patterns of eCB-related genes and applied pharmacological

41 agents to examine how the eCB system impacts glial reactivity and the capacity of MG

42 to become Müller glia-derived progenitor cells (MGPCs). We probed single cell RNA-

43 seq libraries to identify eCB-related genes and identify cells with dynamic patterns of

44 expression in damaged retinas. MG and inner retinal neurons expressed the eCB

45 receptor $C N R 1$, as well as enzymes involved in eCB metabolism. In the chick,

46 intraocular injections of 2-Arachidonoylglycerol (2-AG) and Anandamide (AEA)

47 potentiated the formation of MGPCs. Consistent with these findings, CNR1-agonists

48 and MGLL-inhibitor promoted reprogramming, whereas CNR1-antagonist and inhibitors

49 of eCB synthesis suppressed reprogramming. Surprisingly, retinal microglia were

50 largely unaffected by increases or decreases in eCB signaling in both chick and mouse

51 models. However, eCB-signaling suppressed the activation of NFkB-reporter in MG in

52 damaged mouse retinas. We conclude that the eCB system in the retina influences the 53 reactivity of MG and is important for regulating glial reactivity and the reprogramming of 
54 MG into proliferating MGPCs, but not for regulating the reactivity of immune cells in the

55 retina.

Keywords: Endocannabinoids, Müller glia, Müller glia derived progenitor cells,

57 scRNA-seq, Müller glia reprogramming

\section{Main Points}

60 Müller glia express CNR1 receptor and endocannabinoid synthesis genes.

61 Endocannabinoids after retinal damage promote the formation of Müller glia derived

62 progenitor cells in chick.

63 Endocannabinoids reduce NFkB activity in mouse Müller glia.

\section{Introduction}

The endocannabinoid (eCB) system has been well-studied in the visual system

67 and is known to modulate physiologic functions in different ocular tissues, including the

68 retina (reviewed by (Schwitzer et al., 2016). The eCB system consists of cannabinoid

69 receptors 1 and 2 (CNR1, CNR2), endogenous ligands 2-Arachidonoylglycerol (2-AG)

70 and Arachidonoylethanolamide (AEA), and the enzymes that control ligand synthesis

71 and degradation. The eCB pathway has been identified in the retinas of different

72 vertebrates including embryonic chick (da Silva Sampaio et al., 2018), goldfish (Yazulla

73 et al., 2000), rat (Yang et al., 2016), bovine (Bisogno et al., 1999), porcine (Matsuda et

74 al., 1997), mouse (Hu et al., 2010), and human (Straiker et al., 1999). The expression of

75 CNR1 and CNR2 receptors in the central nervous system varies across species but

76 typically includes distinct types of neurons, astrocytes, microglia, and Müller glia.

77 Activation of eCB receptors is known to modulate neurotransmission (Diana and 
78 Bregestovski, 2005), synaptic plasticity (Xu and Chen, 2015), neuroinflammation

79 (Centonze et al., 2007), and neuroprotection (Slusar et al., 2013).

Müller glia (MG) are thought to play a role in regulating eCBs in the retina. Both

81 CNR1 and CNR2 receptors have been identified in goldfish MG (Yazulla et al., 2000),

82 and CNR2 receptors have been identified in the retinas of vervet monkeys (Bouskila et

83 al., 2013). eCBs have been shown to modify activity or suppress T-type voltage gated

84 calcium channels in rat MG (Yang et al., 2016) and modulate the inflammatory micro-

85 environment (Silverman and Wong, 2018). MG possess pathogen- and damage-

86 associated molecular pattern (PAMP/DAMP) receptors to respond to pathological

87 conditions (Kumar and Shamsuddin, 2012; Kumar et al., 2013; Shamsuddin and Kumar,

88 2011). Activation leads to the secretion of pro-inflammatory cytokines to facilitate the

89 migration and activation of macrophages and microglia (Inoue et al., 1996). At the same

90 time, retinal microglia become reactive and coordinate inflammation with MG, which

91 results in NF-kB activation, concomitant reactive gliosis, and formation of MGPCs

92 (Palazzo et al., 2019). However, MG also produce anti-inflammatory signals such as

93 TGFB2 (Palazzo et al., 2020) and TIMP3 (Campbell et al., 2019) to suppress

94 inflammation. eCBs are believed to have anti-inflammatory actions within the central

95 nervous system (Nagarkatti et al., 2009). Little is known about how eCBs influence

96 inflammation in the retina and whether eCBs impact the ability of $M G$ to reprogram into

97 MG-derived progenitor cells (MGPCs).

98 The impact of inflammatory signals on MG is context specific, dependent on the

99 combination of cytokines and the model of damage. In zebrafish, TNFa (Iribarne et al.,

100 2019) and IL-6 (Zhao et al., 2014) are necessary for MG to transition to a reactive state 
101 into a proliferating progenitor-like cells. In the chick, by comparison, TNF alone does not 102 induce MGPCs and activation of the NF-kB pathway inhibits the formation of MGPCs

103 (Hoang et al., 2020; Palazzo et al., 2020). When microglia are ablated, MGPCs fail to 104 form (Fischer et al., 2014), and the effects of NF-kB-inhibition are reversed to promote 105 the formation of MGPCs (Palazzo et al., 2020). In damaged mouse retinas, reactive 106 MG rapidly transition into a gliotic state and are forced back into a resting state, in part, 107 by regulatory networks involving NF-kB-related factors (Hoang et al., 2020). This 108 suggests that there is an important balance of inflammatory cytokines and timing of 109 signals to drive the reprogramming of MG to dedifferentiate and proliferate as MGPCs.

110 It is currently thought that rapid induction of microglial reactivity is required to "kick-start"

111 MG reactivity as an initial step of reprogramming (Fischer et al., 2014; White et al., 112 2017), whereas sustained elevated microglial reactivity suppresses the neuronal 113 differentiation of progeny produced by MGPCs (Palazzo et al., 2020; Todd et al., 2020). In this study we investigate how eCBs influence glial reactivity, inflammation, and 115 reprogramming of MG in the chick retina. Using scRNA-seq, we analyze the expression 116 pattern of genes in the eCB system and changes in these genes following retinal 117 damage. We apply pharmacological agents to activate or inhibit eCB-signaling and 118 assess changes in glial activation and reprogramming of MG into proliferating MGPCs.

120 Methods and Materials:

121 Animals:

The animals approved in these experiments followed guidelines established by 123 the National Institutes of Health and IACUC at The Ohio State University. P0 wildtype 
124 leghorn chicks (Gallus gallus domesticus) were obtained from Meyer Hatchery (Polk,

125 Ohio). Post-hatch chicks were housed in stainless-steel brooders at $25^{\circ} \mathrm{C}$ with a diurnal

126 cycle of 12 hours light, 12 hours dark (8:00 AM-8:00 PM) and provided water and

127 Purina $^{\mathrm{tm}}$ chick starter ad libitum.

129 Intraocular injections:

130 Chicks were anesthetized with $2.5 \%$ isoflurane mixed with oxygen from a non-

131 rebreathing vaporizer. The intraocular injections were performed as previously

132 described (Fischer et al., 1998). With all injection paradigms, both pharmacological and

133 vehicle treatments were administered to the right and left eye respectively. Compounds

134 were injected in $20 \mu$ sterile saline with $0.05 \mathrm{mg} / \mathrm{ml}$ bovine serum albumin added as a

135 carrier. Compounds included: NMDA (500nmol dose high dose, 60nmol low dose;

136 Sigma-Aldrich), JJKK048 (0.25mg/dose Sigma-Aldrich), ARN19874 (0.25mg/dose

137 AOBIOUS), rimonabant (0.25mg/dose Sigma-Aldrich), PF 04457845 (0.25mg/dose

138 Sigma-Aldrich), Orlistat (0.25mg/dose Sigma-Aldrich), URB 597 (0.25mg/dose Sigma-

139 Aldrich). 5-Ethynyl-2'-deoxyuridine (EdU) was intravitreally injected to label the nuclei of

140 proliferating cells. Injection paradigms are included in each figure.

Enzyme-linked Immunosorbent Assay

143 Endocannabinoids were extracted from retinal tissue and screened for 2-AG

144 levels using a direct competitive enzyme linked immunosorbent assay (MyBioSource).

145 Three retinas were extracted from each treatment group and placed in 5:3

146 homogenization solution (formic acid $\mathrm{pH}=3$ ): extraction solution (9:1 
147 ethylacetate:hexane) on ice. The tissue was homogenized with high intensity sonication

148 on ice, frozen at -20 , and the nonaqueous fraction was removed for evaporation and

149 rehydration in DMSO. The lipid extract was applied to the wells of ELISA and the

150 protocol was followed per the manufacturer's instructions.

Single Cell RNA sequencing of retinas

Retinas were obtained from postnatal chick and adult mice. Isolated retinas were dissociated in a $0.25 \%$ papain solution in Hank's balanced salt solution (HBSS), $\mathrm{pH}=$

1557.4 , for 30 minutes, and suspensions were frequently triturated. The dissociated cells were passed through a sterile $70 \mu \mathrm{m}$ filter to remove large particulate debris. Dissociated

157 cells were assessed for viability (Countess II; Invitrogen) and cell-density diluted to 700 cell/ $\mu$ l. Each single cell cDNA library was prepared for a target of 10,000 cells per sample. The cell suspension and Chromium Single Cell 3' V3 reagents (10X Genomics) were loaded onto chips to capture individual cells with individual gel beads in emulsion (GEMs) using 10X Chromium Controller. cDNA and library amplification for an optimal signal was 12 and 10 cycles respectively. Samples were multiplexed for sequencing on Illumina's Novaseq6000 (Novogene). Sequencer files were converted from a BCL to a

164 Fastq format, where the sequence files were de-multiplexed, aligned, and annotated using the chick ENSMBL database (GRCg6a, Ensembl release 94) and Cell Ranger software (10x Genomics). Using Seurat toolkits, Uniform Manifold Approximation and

167 Projection for Dimension Reduction (UMAP) plots were generated from aggregates of

168 multiple scRNA-seq libraries (Butler et al., 2018; Satija et al., 2015). Compiled in each

169 UMAP plot are two biological library replicates for each experimental condition. Seurat 
was used to construct violin/scatter plots. Significance of difference in violin/scatter plots

171 was determined using a Wilcoxon Rank Sum test with Bonferroni correction. Genes that

172 were used to identify different types of retinal cells included the following: (1) Müller glia:

173 GLUL, VIM, SCL1A3, RLBP1, (2) MGPCs: PCNA, CDK1, TOP2A, ASCL1, (3) microglia:

174 C1QA, C1QB, CCL4, CSF1R, TMEM22, (4) ganglion cells: THY1, POU4F2, RBPMS2,

175 NEFL, NEFM, (5) amacrine cells: GAD67, CALB2, TFAP2A, (6) horizontal cells:

176 PROX1, CALB2, NTRK1, (7) bipolar cells: VSX1, OTX2, GRIK1, GABRA1, and (7) cone

177 photoreceptors: CALB1, GNAT2, OPN1LW, and (8) rod photoreceptors: RHO, NR2E3,

178 ARR3. scRNA-seq libraries can be queried at:

179 https://proteinpaint.stjude.org/F/2019.retina.scRNA.html

Fixation, sectioning, and immunocytochemistry: described previously (Fischer et al., 2008, 2009a). Dilutions and commercial sources of antibodies used in this study are listed in table 2. Labeling was not due to non-specific labeling of secondary antibodies or tissue autofluorescence because sections incubated with secondary antibodies alone were devoid of fluorescence. Secondary antibodies included donkey-anti-goat-Alexa488/568, goat-anti-rabbit-Alexa488/568/647, goat-anti188 mouse-Alexa488/568/647, goat-anti-rat-Alexa488 (Life Technologies) diluted to 1:1000 in PBS and $0.2 \%$ Triton X-100.

Labeling for EdU: 
194 Samples were washed for 5 minutes with PBS, permeabilized with 0.5\% Triton X-100 in

195 PBS for 1 minute at room temperature and washed twice for 5 minutes in PBS. Sections

196 were incubated for 30 minutes at room temperature in a buffer consisting of $100 \mathrm{mM}$

197 Tris, $8 \mathrm{mM} \mathrm{CuSO}_{4}$, and $100 \mathrm{mM}$ ascorbic acid in $\mathrm{dH}_{2} \mathrm{O}$. The Alexa Fluor 568 Azide

198 (Thermo Fisher Scientific) was added to the buffer at a 1:100 dilution.

Terminal deoxynucleotidyl transferase dUTP nick end labeling (TUNEL):

The TUNEL assay was implemented to identify dying cells by imaging

202 fluorescent labeling of double stranded DNA breaks in nuclei. The In Situ Cell Death Kit

203 (TMR red; Roche Applied Science) was applied to fixed retinal sections as per the

204 manufacturer's instructions.

Photography, measurements, cell counts and statistics:

Microscopy images of retinal sections were captured with the Leica DM5000B

208 microscope with epifluorescence and the Leica DC500 digital camera. High resolution

209 confocal images were obtained with a Leica SP8 available in The Department of

210 Neuroscience Imaging Facility at The Ohio State University. Representative images are

211 modified to have enhanced color, brightness, and contrast for improved clarity using

212 Adobe Photoshop. In EdU proliferation assays, a fixed region of retina was counted and

213 average numbers of Sox2 and EdU co-labeled cells. The retinal region selected for

214 investigation was standardized between treatment and control groups to reduce

215 variability and improve reproducibility. 
Similar to previous reports (Fischer et al., 2009b, 2009c; Ghai et al., 2009),

217 immunofluorescence was quantified by using Image $\mathrm{J}(\mathrm{NIH})$. Identical illumination,

218 microscope, and camera settings were used to obtain images for quantification. Retinal

219 areas were sampled from 5.4 MP digital images. These areas were randomly sampled

220 over the inner nuclear layer (INL) where the nuclei of the bipolar and amacrine neurons

221 were observed. Measurements of immunofluorescence were performed using ImagePro

2226.2 as described previously (Ghai et al., 2009; Stanke et al., 2010; Todd and Fischer,

223 2015). The density sum was calculated as the total of pixel values for all pixels within

224 thresholded regions. The mean density sum was calculated for the pixels within

225 threshold regions from $\geq 5$ retinas for each experimental condition. GraphPad Prism 6

226 was used for statistical analyses.

Measurements of immunofluorescence of CD45 in microglia were made from

228 single optical confocal sections by selecting the total area of pixel values above

229 threshold $(\geq 70)$ for CD45 immunofluorescence. Measurements were made for regions

230 containing pixels with intensity values of 70 or greater $(0 \square=\square$ black and

$231255 \square=\square$ saturated). The total area was calculated for regions with pixel intensities above

232 threshold. The intensity sum was calculated as the total of pixel values for all pixels

233 within threshold regions. The mean intensity sum was calculated for the pixels within

234 threshold regions from $\geq 5$ retinas for each experimental condition. For characterization

235 of the morphology of the individual microglia, a Sholl analysis was used to characterize

236 the size, sphericity, and projections (ImageJ).

237 For statistical evaluation of differences across treatments, a two-tailed paired $t$ -

238 test was applied for intra-individual variability where each biological sample also served 
239 as its own control. For two treatment groups comparing inter-individual variability, a two-

240 tailed unpaired $t$-test was applied. For multivariate analysis, an ANOVA with the

241 associated Tukey Test was used to evaluate any significant differences between

242 multiple groups.

247 Results:

\section{Patterns of expression of eCB-related genes} scRNA-seq libraries were aggregated from control retinas and retinas treated

250 with NMDA-damage at different times (3, 12 and $48 \mathrm{hrs}$ ) after treatment. These libraries

251 were clustered and analyzed for expression eCB-related genes under MG-

252 reprogramming conditions (Fig. 1). UMAP plots were generated and the identity of

253 clusters of cells established based on expression of cell-distinguishing markers (Fig.

254 1b,c). Resting MG occupied a discrete cluster of cells and expressed high levels of 255 GLUL, VIM (Fig. 1d), RLBP1 and CA2 (supplemental Fig. 1a-e,i). After damage, MG 256 down-regulate these genes during transition to a reactive phenotype and up-regulate

257 markers associated reactivity such as MDK, HBEGF, MANF (Fig. 1e, supplemental Fig.

258 1e,f,i), with some genes such as TGFB2, ATF3 and TNFRSF1A upregulated with 3hrs

259 of NMDA-treatment (supplemental Fig. 1f,g,i). Upregulation of progenitor- and

260 proliferation-related genes was observed in MGPCs at 48hrs after NMDA-treatment 
261 (supplemental Fig. 1c,h,i), consistent with prior reports (Hoang et al., 2020; Campbell et al., 2021,).

The expression of eCB-related genes in MG has been previously reported in

264 developing chick retina (da Silva Sampaio et al., 2018). The eCB system includes receptors $C N R 1$ and $C N R 2$ and enzymes involved in the synthesis (NAPEPLD, DAGLA and $D A G L B$ ) and degradation (FAAH and MGLL) of 2-AG and AEA (Fig. 1a). We detected CNR1, MGLL, DAGLA, DAGLB, NAPELPD and FAAH in control retinas and at different times after NMDA-treatment (Fig. 1f-i). CNR2 was not detected. MGLL was prevalent and highly expressed by resting MG, but down-regulated in activated MG (Fig. $1 \mathrm{~g}, \mathrm{f})$. By comparison, levels of expression and prevalence of CNR1 was high in many

271 amacrine cells, and in a few ganglion and bipolar cells (Fig. 1f). MGLL and NAPEPLD

272 were detected at high levels in many microglia, NIRG cells and bipolar cells, and in

273 relatively few photoreceptors, ganglion and horizontal cells (Fig. 1e). DAGLA and FAAH

274 had scattered expression across many retinal cell types, whereas DAGLB was

275 prominently expressed at high levels in photoreceptors and inner retinal neurons (Fig.

276 1h). CNR1 and eCB-related genes, except $F A A H$, were uniformly down-regulated in

277 levels, but increased in prevalence in activated MG after NMDA-treatment (Fig. 1g-i).

278 To directly compare expression levels in MG across different treatment

279 paradigms we isolated and re-aggregated MG from different treatment groups, including

280 retinas treated with the combination of NMDA+insulin+FGF2, NMDA alone and

281 insulin+FGF2 alone. Resting MG, activated MG from 24 hrs after NMDA-treatment and

2822 doses of insulin+FGF2 formed distinct clusters of cells (Fig. 2a,b). Further, MGPCs

283 formed discrete regions of cells wherein cell cycle progression formed the basis of 
284 spatial segregation with the majority of cells progressing through the cell cycle from 285 retinas at $72 \mathrm{hrs}$ after NMDA or NMDA+FGF2+insulin treatment (Fig. 2c,e). The largest

286 increase in levels and prevalence of expression of $C N R 1$ was observed in reactive MG 287 from 48+72hrs after NMDA (Fig. 2f,g). By comparison, the largest decrease in levels 288 and prevalence of expression of MGLL, DAGLA, DAGLB and NAPEPLD were observed 289 for MG clustered among treatments with insulin+FGF2 and in the MGPC3 cluster (Fig.

$2902 \mathrm{f}, \mathrm{g})$. Collectively, these findings suggest that the expression of CNR1 by MG is

291 changed in response to neuronal damage, whereas levels of MGLL and other eCB292 related genes were down-regulated by treatment with NMDA (neuronal damage) or 293 insulin and FGF2 (no neuronal damage).

\section{eCBs promote the formation of MGPCs after damage}

Although patterns of gene expression can be complex and context dependent, dynamic changes in mRNA levels are strongly correlated with changes in protein levels

298 and function (Liu et al., 2016). Accordingly, we tested activation of eCB-signaling 299 influence glial reactivity, neuronal survival and the formation of MGPCs. The ligand 300 binding affinity of chick CNR receptors remains uncertain. Thus, 2-AG and AEA were 301 co-injected to maximize the probability of activation of CNR1 receptors. We tested 302 whether co-injection of 2-AG and AEA influenced the formation of proliferating MGPCs.

303 Compared to numbers of proliferating MGPCs in NMDA-damaged retinas, treatment 304 with eCBs resulted in a significant increase in numbers of Sox2/EdU-positive MGPCs

305 (Fig. 3a,b). Consistent with these findings, numbers of proliferating MGPCs that 306 expressed neurofilament and phospho-histone $\mathrm{H} 3(\mathrm{pHH} 3)$ were significantly increased 
by treatment with 2-AG and AEA (Fig. 3c,d). Levels of retinal damage influence the reprogramming of MG; there is a positive correlation between numbers of dying cells and numbers of proliferating MGPCs (Fischer and Reh, 2001; Fischer et al., 2004).

311 the TUNEL method. The number of TUNEL-positive cells was unchanged by 2-AG and

312 AEA, suggesting that levels of cell death in NMDA-damaged retinas were unaffected by 313 addition of eCBs (Fig 3e,f).

Targeting the еCB synthesis and degradation influences MG reprogramming retinas, we investigated whether levels of eCBs were influenced by damage or drugs

318 that interfere with synthesis or degradation of AEA and 2-AG. We applied Orlistat, an inhibitor of DAGL, to reduce eCB synthesis and JJKK-048, an inhibitor to MGLL, to suppress eCB degradation (Hillard, 2015). By using competitive inhibition ELISAs, we measured levels 2-AG and AEA in retinas treated with NMDA and inhibitors. We detected low levels of 2-AG in the retina, that did not significantly change with NMDA damage at 72 hours (Fig. 4a). Although we failed to detect a significant change in 2-AG with Orlistat treatment, injections of JJKK-048 resulted in a significant increase in retinal

325 levels of 2-AG (Fig. 4a). AEA was not detectable within the threshold range of the 326 ELISA; thus, inhibitor treatments had no detectable impact on levels of AEA (Fig. 4b).

327 Since levels of AEA fell below levels of detection we did not probe for changes in AEAlevels following treatment with inhibitors of NAPEPLD or FAAH. 
331 CNR1 receptor with a small molecule agonist and an antagonist. Win-55, 212-2

332 (Win55) is a potent CNR agonist in humans, mice and chickens (Stincic and Hyson,

333 2011). Rimonabant is a potent and selective antagonist that inhibits CNR1-mediated

334 cell-signaling (Ádám et al., 2008) (Hillard, 2015). Activation of CNR1 with Win55

335 increased numbers of proliferating MGPCs, whereas inhibition of CNR1 with rimonabant

336 had the opposite effect (Fig. 4c,d,e). MGLL inhibitor (JJKK048), which increased levels

337 of 2-AG (Fig. 4a), increased numbers of proliferating of MGPCs (Fig. 4f). By

338 comparison, the DAGL inhibitor Orlistat significantly decreased numbers of MGPCs (Fig

$3394 \mathrm{~g})$. Overall, treatments expected to increase eCB-signaling increased MG

340 reprogramming and treatments to decrease eCB-signaling decreased MG

341 reprogramming.

We next targeted enzymes that influence the synthesis (NAPEPLD) or

343 degradation (FAAH) of AEA. Inhibition of NAPELPD with ARN19784 had no effect upon

344 numbers of proliferating MGPCs (Fig. 5a,b), whereas numbers of proliferating microglia

345 were increased (Fig 5c,d) and numbers of proliferating NIRG cells and dying cells were

346 decreased (Fig. 5e-h). By comparison, inhibition of FAAH with URB597 or PF-044 had

347 no significant effect upon proliferating MGPCs, microglia and NIRG cells, or cell death

348 (Fig. 5d,f,h). We bioinformatically isolated scRNA-seq data for microglia and performed

349 a fine-grain analysis. Microglia formed discrete UMAP clustering of resting and

350 activated microglia from control retinas and retinas at 3, 12 and 48 hrs after NMDA-

351 treatment (Fig. 5i-I). We detected scattered expression of relatively high levels of $M G L L$

352 and NAPEPLD, but no expression of $C N R 1$ (Fig. $5 \mathrm{~m}$ ) or FAAH (not shown). 
Collectively, these data suggests that cells in chick retina support production of

354 2-AG over AEA in the context of damage and reprogramming. Further the reactivity of

355 some microglia and NIRG cells, but not MG, is influenced by inhibition of NAPEPLD,

356 and these responses are consistent with patterns of expression seen in scRNA-seq

357 databases.

\section{Microglia Reactivity and eCBs}

Retinal microglia serve homeostatic functions and mediate inflammation in response to damage and pathogens (Silverman and Wong, 2018). In response to excitotoxic damage in the chick, the microglia become reactive, leading to accumulation of monocytes, proliferation, and upregulation of inflammatory cytokines (Fischer et al., 2014). Given the known association of microglia, inflammation and eCB-signaling (Stella, 2009) and the dependence of MGPC formation on signals provided by reactive microglial (Fischer et al., 2014; Palazzo et al., 2020), we investigated the impact of drugs targeting CNR1 and 2-AG metabolism on microglia reactivity, proliferation and 368 reactivity.

Microglia are sparsely distributed and highly ramified when quiescent, but

370 become reactive and transiently accumulate after NMDA-treatment (Fischer et al.,

371 2014). We applied established metrics of microglia reactivity in the chick model (Gallina,

372 2015), including microglia infiltration/accumulation, proliferation, CD45-intensity, cell

373 area, and ramification were compared in different eCB targeted treatments. eCBs and

374 small molecule inhibitors had no significant effect on the reactivity of microglia in

375 damaged retinas (Fig. 6). Both the small molecule drugs and 2-AG/AEA did not 
376 influence total numbers of $\mathrm{CD} 45^{+}$cells compared to damage alone (Fig. 6a-c). Similarly,

377 the number of proliferating $\mathrm{CD} 45^{+}$cells was unaffected by eCB treatments in damaged

378 retinas (Fig. 5a-c). Similarly, the area and intensity of $\mathrm{CD} 45^{+}$immunolabeling were

379 unaffected by drugs targeting eCBs (Fig. 6c). Using a Sholl analysis to quantify

380 microglia shape, we quantified the maximum intersections (ramification index), mean

381 intersections (centroid value), and maximum intersection radius (processes distribution).

382 Although the morphology of resting microglia in saline treated retinas was significantly

383 different from the morphology of microglia in NMDA-damaged retinas (Fig. 6d), the

384 morphology of reactive microglia was unchanged by drugs targeting eCB receptors or

385 metabolic enzymes (table 1).

\section{NF-kB activation is reduced in mouse MG when promoting eCB signaling}

$\mathrm{NF}-\mathrm{kB}$ is a transcription factor known to be a primary transductor of the innate

and adaptive immunity, and a central mediator of inflammatory responses to pathogens

or tissue damage (Liu et al., 2017). In the chick, activation or inhibition of the NF-kB

391 pathway has a significant impact on the ability of MG to become proliferating MGPCs

392 (Palazzo et al., 2020). In the mouse retina, NF-kB-signaling has been implicated a

393 signaling "hub" that may act to drive MG into a reactive state and then back into a

394 resting state (Hoang et al., 2020). Accordingly, we investigated the anti-inflammatory

395 properties of eCBs using the NF-kB reporter in the mouse retina. We used the mouse

396 model because there are no cell-level read-outs of NF-kB-signaling available in the

397 chick (Palazzo et al., 2020). 
We first assessed the patterns of expression of eCB-related factors in normal and NMDA-damaged mouse retinas in aggregated scRNA-seq libraries. UMAP analysis of cells from control and damaged retinas revealed discrete clusters of cell types (Fig. 7a). Neurons from control and damaged retinas were clustered together regardless of time after NMDA-treatment (Fig. 7a). By contrast, resting MG, including MG from 48 to $72 \mathrm{hr}$ after NMDA, and activated MG from 3, 6, 12, and $24 \mathrm{hr}$ after treatment were spatially separated across the UMAP plot (Fig. 7c). Consistent with previous reports (Bouchard et al., 2015), Cnr1 was detected in amacrine and ganglion cells (Fig. 7d), whereas Cnr2 was not detected at significant levels in any retinal cells (not shown). By comparison, Daglb was detected in many retinal neurons including photoreceptors and

408 bipolar cells, and Mgll was detected prominently in ganglion cells, glycinergic amacrine 409 cells, and resting MG (Fig. 7e), similar to patterns seen in chick retinas (Fig. 1). Dagla

410 was not detected (not shown). Although Napepld was not widely expressed, Faah had

411 scattered expression in bipolar cells, and rod and cone photoreceptors (Fig. 7f). We utilized the cis-NF-kB ${ }^{\text {eGFP }}$ reporter mouse line to visualize cells where p65 is 413 driving transcription as a read-out of NF-kB-signaling (Magness et al., 2004). In 414 undamaged retinas, NFkB reporter was observed in a few endothelial cell whereas 415 eGFP reporter was not detected in any retinal neurons or glia (Fig. 8a). At 48hrs after 416 NMDA damage significant numbers of MG express NFkB-eGFP (Fig. 8a,b). Treatment 417 with CNR1 agonist Win55 or eCBs (2-AG/AEA) resulted in a significant reduction in 418 numbers of MG that were eGFP-positive (Fig. 8a,c). By contrast, treatment with CNR1 419 antagonist (Rimonabant) significantly increased numbes of eGFP-positive MG (Fig. 420 8a,c). To determine if changes in cell death were influenced eCBs we performed 
421 TUNEL staining. There was no change in cell death in retinas treated with eCBs,

422 Rimonabant or Win55 (Fig. 8d,e). In addition, there was there was no obvious change

423 in microglial morphology (Fig. 8f) and no significant change in the accumulation of

424 microglia in damaged retinas treated with eCBs, Rimonabant or Win55 (Fig. 8g). These

425 data suggest that eCBs influence NF-kB signaling in mammalian MG, whereas

426 neuroprotection and microglial reactivity are unaffected.

428 Discussion:

429 In this study we investigated the roles of eCB-signaling in the chick model of MG 430 reprogramming. Retinal cells widely expressed both $C N R 1$ and genes involved in the

431 synthesis and degradation of eCBs. The levels of expression and proportion of MG that 432 express these genes significantly change following damage and during the transition to 433 a proliferating progenitor-like cell. These changes in expression imply functions for 434 eCBs in damaged retinas and during the formation of MGPCs. Indeed, we found that 435 reprogramming of MG into proliferating MGPCs was promoted by eCBs and by CNR1 436 agonists or enzyme inhibitor that increase retinal levels of 2-AG. Microglia maintained a 437 reactive phenotype in damaged retinas regardless of treatment with eCB drugs. These 438 findings support recent reports that the inflammatory state of MG is important to the 439 transition from resting to reactive, and then to a progenitor-like cell (Fischer et al., 2014;

440 Hoang et al., 2020; White et al., 2017). 
In the embryonic chick retina the expression of CNR1 and MGLL has been

444 reported in MG (da Silva Sampaio et al., 2018). In addition to expression in MG, we

445 detected CNR1 in MG and in a population of amacrine cells and MGLL was detected in

446 MG, some types of ganglion cells and oligodendrocytes. The eCB-related genes were

447 present in MG but at low levels in a small proportion of MG. This pattern of expression

448 is in contrast with high-expressing glial markers, such as glutamine synthetase (GLUL),

449 retinaldehyde binding protein $1(R L B P 1)$ and carbonic anhydrase $2(C A 2)$ that are

450 detected in $>96 \%$ of MG in scRNA-seq preparations (Campbell et al., 2021; Palazzo et

451 al., 2020). We believe this may be due to sensitivity limitations of the reagents wherein

452 low-copy transcripts may not be readily detected. It is also possible that a sub-

453 population of MG express eCB-related genes, suggesting heterogeneity among MG

454 types. However, the eCB-expressing MG subsets are scattered homogenously in these

455 clusters and do not correlate with unique markers corresponding to biologically unique

456 subclusters.

458 Elevated eCBs promote $M G$ reprogramming

459 Although the roles of eCBs have been investigated in the visual system, little is

460 known about how eCBs influence MG reprogramming in different models of retinal

461 regeneration. We observed that exogenous eCB increased the proportion of MG that

462 formed proliferating MGPCs. This effect was reproduced by inhibition of MGLL with

463 JJKK048 which is expected to increase retinal levels of 2-AG. Similarly, this drug has

464 been validated to target MGLL and increase levels of 2-AG levels in mice (Hillard,

465 2015). Orlistat has been shown to inhibit DAGL and suppress 2-AG synthesis in 
466 humans (Bisogno et al., 2006). Although we observed a decrease in number of

467 proliferating MGPCs with Orlistat treatment, we did not observe a significant decrease in

468 2-AG as measured by ELISA. This may have resulted from the low sensitivity threshold

469 for detecting 2-AG. These lipids represent a very small fraction of total lipids from

470 whole-retina extracts. Alternatively, Orlistat could be targeting fatty acid synthase

471 (FASN), disrupting lipid metabolism to influence retinal levels of 2-AG (Kridel et al., 472 2004).

We examined whether CNR1 may have mediated eCB effects applying selective

474 agonists and antagonists, drugs with validated specificity in the chick CNS (Ádám et al.,

475 2008; Stincic and Hyson, 2011). We observed complimentary effects with Win55 (CNR1

476 agonist) increasing and rimonabant (CNR1 antagonist) decreasing numbers of

477 proliferating MGPCs. Nevertheless, we cannot exclude the possibility that these effects

478 are due to indirect actions at MG given that amacrine and ganglion cells express

479 significant levels of CNR1 and may have mediated effects on MGPCs through

480 secondary factors. We failed to detect CNR1 expression among microglia, NIRG cells or 481 oligodendrocytes in normal retinas or after NMDA-treatment.

483 eCBs. In different animal models and cell types changes in cell physiology are mediated 484 via interactions and cross-talk with other cell-signaling pathways, such as Notch1

485 (Frampton et al., 2010), mTor (Palazuelos et al., 2012), MAPK/PI-3K (Dalton et al., 486 2009), and Wnt signaling (Nalli et al., 2019). These cell-signaling pathways are known

487 to be active and promote the reprogramming of MG into MGPCs in the chick model

488 (Fischer et al., 2002; Gallina et al., 2016; Ghai et al., 2010; Zelinka et al., 2016). 
489 However, we have yet to identify the interactions between eCB-signaling and other cell-

490 signaling pathways that have been implicated in the reprogramming of MG. These

491 connections may be difficult to identify in undamaged retinas given that homeostatic

492 enzymes reduce eCB levels and because the sensitivity of MG to eCBs may increase

493 after damage with increased prevalence of CNR1-expression among MG.

eCBs are not neuroprotective to excitotoxic NMDA damage eCBs have been shown to provide neuroprotection in degenerative retinal diseases (Rapino et al., 2018). Recent articles have even suggested that 2-AG can mediate neuroprotection against AMPA toxicity in the rat retina (Kokona et al., 2021).

499 We investigated eCB-related neuroprotection because levels of retinal damage and cell

500 death are known to influence the reprogramming of MG in to MGPCs. Although,

501 injections of 2-AG and AEA did not impact numbers of dying cells, the CNR1 agonist

502 Win55 increased cell death in the chick retina (supplemental Fig. 2a,b). This could result

503 from interactions with ion channels that are known to occur with these lipophilic eCB

504 ligands (Pertwee, 2010). Alternatively, differences in excitotoxicity with eCB

505 administration could be due to targeting NMDA vs AMPA receptors in the damage

506 model. (2021) reported the death of photoreceptors with AMPA-selective agonists,

507 which does not occur with NMDA. In other disease models where 2-AG provides

508 neuroprotection the mode of cellular damage is slow and progressive (Centonze et al.,

509 2007), unlike our model of NMDA-induced excitotoxicity which acute and severe.

511 Microglia reactivity is not influenced by eCBs 
eCBs are believed to be potent anti-inflammatory drugs in the CNS (Ullrich et al.,

513 2007). This is frequently suggested as mechanism of clinical benefit in pathological

514 states. In the chick model of reprogramming, we have used dexamethasone GCR

515 receptor agonist to repress the reactivity of microglia (Gallina, 2015). Similarly, treating

516 damaged retinas with NF-kB inhibitor sulfasalazine also resulted in a decrease in the

517 reactive proliferation of $\mathrm{CD}^{+} 5^{+}$cells (Palazzo et al., 2020). With eCBs and small

518 molecule drugs, there was no evidence that the reactivity of the microglia was

519 influenced. Studies have demonstrated that reduced accumulation of reactive microglia

520 results in neuroprotective effects whereas increased accumulation of microglial

521 reactivity can be detrimental to neuronal survival (Fischer et al., 2015; Todd et al., 2019)

522 (Fischer et al., 2105 Glia; Todd et al., $2019 \mathrm{~J}$ Neuroinflam; other refs). In the current

523 study, however, inhibition of NAPEPLD increased the accumulation of reactive

524 microglia, while numbers of dying cells were reduced. This may have resulted from

525 multiple cellular targets being directly affected by the NAPEPLD inhibitor since

526 NAPEPLD was detected in microglia, MG and inner retinal neurons.

527 Our findings are consistent with the notion that eCB-signaling is, in part,

528 manifested through MG. However, we cannot exclude the possibility of eCBs mediate

529 changes in production pro-inflammatory cytokines from reactive microglia in damaged

530 retinas. The relationship between these inflammatory factors and MGPC formation is

531 complex and time-dependent. For example, decreased retinal inflammation from

532 inhibition of microglial reactivity with glucocorticoid agonists reduced MGPC formation,

533 whereas decreased retinal inflammation from inhibition of NFkB-signaling increased

534 MGPC formation (Gallina, 2015; Palazzo et al., 2020). However, the impact of NFkB- 
535 signaling on the formation of MGPCs was reversed when the microglia were ablated

536 (Palazzo et al., 2020). Pro-inflammatory factors likely directly influence MG, with

537 evidence that MG activate NF-kB-signaling and express cytokine receptors in damaged

538 retinas (Palazzo et al., 2020). Further studies are required to determine the impact of

539 pro-inflammatory signals on microglia and MG in eCB-treated retinas to better

540 characterize the coordination between these glial cells.

541

542 eCBs repress NF-kB in mouse MG

543 While reporter lines for MG do not exist in the chick model, the mouse model of

544 retinal damage was applied to the cis-NF-kB ${ }^{\text {eGFP }}$ reporter line to identify cells where 065

545 translocates into the nucleus and drives the expression of the eGFP-reporter. We find

546 that MG are the primary cell type that activates NF-kB-signaling in NMDA-damaged

547 retinas. This supports prior findings in chick that MG respond to proinflammatory

548 cytokines such as TNF associated with NF-kB signaling (Palazzo et al., 2020). After

549 damage eCBs reduced numbers of $\mathrm{GFP}^{+} \mathrm{MG}$, suggesting that eCBs limit the activation

550 of NF-kB in support cells. NF-kB has been implicated as an important pathway in mouse

551 retina that may mediate a switch between reactive gliosis and resting MG (Hoang et al.,

552 2020). Recent studies focused on MG reprogramming have highlighted the importance

553 of the interactions between microglia and MG. The ablation of microglia with CSF1R

554 inhibitor have a dramatic impact on the neurogenic capacity of MG that overexpress

555 Ascl1 (Todd et al., 2020). The absence of microglia induced transcriptomic changes in

556 MG which included the repression of gliosis-associated genes (Todd et al., 2020). The 
557 context, timing and specific cell-signaling pathways that influence the reprogramming

558 capacity of MG in the mammalian retina requires further investigation.

560 Conclusions:

In this study we investigated the impact of eCBs on retinal inflammation and MG

562 reprogramming in the chick model. We found transcriptomic evidence of eCB genes

564 during the transition into MGPCs. Increasing levels of eCBs through intravitreal

565 injections or upregulation of 2-AG via enzyme inhibitors increased numbers of

566 proliferating MGPCs. Surprisingly, cell death and microglia reactivity were largely

567 unaffected by experimental manipulation of levels eCBs in both chick and mouse

568 models of retinal damage. These data support recent evidence that inflammatory

569 signaling play a pivotal role in regulating reactive gliosis, promoting the de-differentiation

570 in MG, and suppressing the neurogenic capacity of MGPCs.

572 Author contributions: WAC - experimental design, execution of experiments,

573 collection of data, data analysis, construction of figures and writing the manuscript. SB

574 and AR - execution of experiments, collection of data and data analysis. TH and SB

575 facilitated the single cell experiments. AJF - experimental design, data analysis,

576 construction of figures and writing the manuscript.

577

578 Competing Interests: The authors have no competing interests to declare. 
580 Data availability: RNA-Seq data are deposited in GitHub

581 https://github.com/jiewwwang/Single-cell-retinal-regeneration

582 https://github.com/fischerlab3140/scRNAseq libraries

583 scRNA-Seq data can be queried at

584 https://proteinpaint.stjude.org/F/2019.retina.scRNA.html. 


\section{References:}

588

Ádám, Á.S., Wenger, T., and Csillag, A. (2008). The cannabinoid CB1 receptor antagonist rimonabant dose-dependently inhibits memory recall in the passive avoidance task in domestic chicks (Gallus domesticus). Brain Research Bulletin 76, 272-274.

Bisogno, T., Delton-Vandenbroucke, I., Milone, A., Lagarde, M., and Di Marzo, V. (1999). Biosynthesis and Inactivation of N-Arachidonoylethanolamine (Anandamide) and $\mathrm{N}$ Docosahexaenoylethanolamine in Bovine Retina. Archives of Biochemistry and Biophysics 370, 300-307.

Bisogno, T., Cascio, M.G., Saha, B., Mahadevan, A., Urbani, P., Minassi, A., Appendino, G., Saturnino, C., Martin, B., Razdan, R., et al. (2006). Development of the first potent and specific inhibitors of endocannabinoid biosynthesis. Biochimica et Biophysica Acta (BBA) - Molecular and Cell Biology of Lipids 1761, 205-212.

Bouchard, J.-F., Casanova, C., Cécyre, B., and Redmond, W.J. (2015). Expression and Function of the Endocannabinoid System in the Retina and the Visual Brain (Hindawi).

Bouskila, J., Javadi, P., Casanova, C., Ptito, M., and Bouchard, J.-F. (2013). Müller cells express the cannabinoid CB2 receptor in the vervet monkey retina. Journal of Comparative Neurology 521, 2399-2415.

Butler, A., Hoffman, P., Smibert, P., Papalexi, E., and Satija, R. (2018). Integrating single-cell transcriptomic data across different conditions, technologies, and species. Nature Biotechnology $36,411-420$.

Campbell, W.A., Deshmukh, A., Blum, S., Todd, L., Mendonca, N., Weist, J., Zent, J., Hoang, T.V., Blackshaw, S., Leight, J., et al. (2019). Matrix-metalloproteinase expression and gelatinase activity in the avian retina and their influence on Müller glia proliferation. Experimental Neurology 320, 112984.

Campbell, W.A., Fritsch-Kelleher, A., Palazzo, I., Hoang, T., Blackshaw, S., and Fischer, A.J. (2021). Midkine is neuroprotective and influences glial reactivity and the formation of Müller gliaderived progenitor cells in chick and mouse retinas. Glia n/a, 1-25.

Centonze, D., Finazzi-Agrò, A., Bernardi, G., and Maccarrone, M. (2007). The endocannabinoid system in targeting inflammatory neurodegenerative diseases. Trends in Pharmacological Sciences 28, 180-187.

Dalton, G.D., Bass, C.E., Van Horn, C., and Howlett, A.C. (2009). Signal Transduction via Cannabinoid Receptors. CNS Neurol Disord Drug Targets 8, 422-431.

Diana, M.A., and Bregestovski, P. (2005). Calcium and endocannabinoids in the modulation of inhibitory synaptic transmission. Cell Calcium 37, 497-505.

Fischer, A.J., and Reh, T.A. (2001). Muller glia are a potential source of neural regeneration in the postnatal chicken retina. Nat Neurosci 4, 247-252. 
623

624

625

626

627

628

629

630

631

632

633

634

635

636

637

638

639

640

641

642

643

644

645

646

647

648

649

650

651

652

653

654

655

656

657

658

659

660

Fischer, A.J., Seltner, R.L.P., Poon, J., and Stell, W.K. (1998). Immunocytochemical characterization of quisqualic acid- and N-methyl-D-aspartate-induced excitotoxicity in the retina of chicks. Journal of Comparative Neurology 393, 1-15.

Fischer, A.J., McGuire, C.R., Dierks, B.D., and Reh, T.A. (2002). Insulin and Fibroblast Growth Factor 2 Activate a Neurogenic Program in Müller Glia of the Chicken Retina. J. Neurosci. 22, 9387-9398.

Fischer, A.J., Schmidt, M., Omar, G., and Reh, T.A. (2004). BMP4 and CNTF are neuroprotective and suppress damage-induced proliferation of Muller glia in the retina. Mol Cell Neurosci 27, 531-542.

Fischer, A.J., Foster, S., Scott, M.A., and Sherwood, P. (2008). The transient expression of LIMdomain transcription factors is coincident with the delayed maturation of photoreceptors in the chicken retina. J Comp Neurol 506, 584-603.

Fischer, A.J., Scott, M.A., Ritchey, E.R., and Sherwood, P. (2009a). Mitogen-activated protein kinase-signaling regulates the ability of Müller glia to proliferate and protect retinal neurons against excitotoxicity. Glia 57, 1538-1552.

Fischer, A.J., Scott, M.A., Ritchey, E.R., and Sherwood, P. (2009b). Mitogen-activated protein kinase-signaling regulates the ability of Müller glia to proliferate and protect retinal neurons against excitotoxicity. Glia 57, 1538-1552.

Fischer, A.J., Scott, M.A., and Tuten, W. (2009c). Mitogen-activated protein kinase-signaling stimulates Muller glia to proliferate in acutely damaged chicken retina. Glia 57, 166-181.

Fischer, A.J., Zelinka, C., Gallina, D., Scott, M.A., and Todd, L. (2014). Reactive microglia and macrophage facilitate the formation of Müller glia-derived retinal progenitors. Glia 62, 16081628.

Fischer, A.J., Zelinka, C., and Milani-Nejad, N. (2015). Reactive retinal microglia, neuronal survival, and the formation of retinal folds and detachments. Glia 63, 313-327.

Frampton, G., Coufal, M., Li, H., Ramirez, J., and DeMorrow, S. (2010). Opposing actions of endocannabinoids on cholangiocarcinoma growth is via the differential activation of Notch signaling. Exp Cell Res 316, 1465-1478.

Gallina, D.Z., C.P. Cebulla, C.M. Fischer, A.J. (2015). Activation of glucocorticoid receptors in Müller glia is protective to retinal neurons and suppresses microglial reactivity. Exp Neurol 273, 114-125.

Gallina, D., Palazzo, I., Steffenson, L., Todd, L., and Fischer, A.J. (2016). Wnt/ß-cateninsignaling and the formation of Müller glia-derived progenitors in the chick retina. Developmental Neurobiology 76, 983-1002.

Ghai, K., Zelinka, C., and Fischer, A.J. (2009). Serotonin released from amacrine neurons is scavenged and degraded in bipolar neurons in the retina. J Neurochem 111, 1-14.

Ghai, K., Zelinka, C., and Fischer, A.J. (2010). Notch signaling influences neuroprotective and proliferative properties of mature Muller glia. J Neurosci 30, 3101-3112. 
Hillard, C.J. (2015). The Endocannabinoid Signaling System in the CNS. In International Review of Neurobiology, (Elsevier), pp. 1-47.

Hoang, T., Wang, J., Boyd, P., Wang, F., Santiago, C., Jiang, L., Yoo, S., Lahne, M., Todd, L.J., Jia, M., et al. (2020). Gene regulatory networks controlling vertebrate retinal regeneration. Science 370 . and Straiker, A. (2010). Architecture of cannabinoid signaling in mouse retina. J Comp Neurol $518,3848-3866$.

Inoue, M., Nakayama, C., and Noguchi, H. (1996). Activating mechanism of CNTF and related cytokines. Mol Neurobiol 12, 195-209.

671 Iribarne, M., Hyde, D.R., and Masai, I. (2019). TNFa Induces Müller Glia to Transition From 672 Non-proliferative Gliosis to a Regenerative Response in Mutant Zebrafish Presenting Chronic 673 Photoreceptor Degeneration. Front. Cell Dev. Biol. 7.

674 Kokona, D., Spyridakos, D., Tzatzarakis, M., Papadogkonaki, S., Filidou, E., Arvanitidis, K.I., arachidonoylglycerol and dual ABHD6/MAGL enzyme inhibitors display neuroprotective and anti-inflammatory actions in the in vivo retinal model of AMPA excitotoxicity. Neuropharmacology 185, 108450.

Kridel, S.J., Axelrod, F., Rozenkrantz, N., and Smith, J.W. (2004). Orlistat Is a Novel Inhibitor of Fatty Acid Synthase with Antitumor Activity. Cancer Res 64, 2070-2075.

Kumar, A., and Shamsuddin, N. (2012). Retinal Muller Glia Initiate Innate Response to Infectious Stimuli via Toll-Like Receptor Signaling. PLOS ONE 7, e29830. Transduct Target Ther 2, 17023. (2004). In vivo pattern of lipopolysaccharide and anti-CD3-induced NF-kappa B activation using a novel gene-targeted enhanced GFP reporter gene mouse. J Immunol 173, 1561-1570. Yamamoto, S. (1997). Metabolism of Anandamide, an Endogenous Cannabinoid Receptor Ligand, in Porcine Ocular Tissues. Experimental Eye Research 64, 707-711. as novel anti-inflammatory drugs. Future Med Chem 1, 1333-1349. 
Palazuelos, J., Ortega, Z., Díaz-Alonso, J., Guzmán, M., and Galve-Roperh, I. (2012). CB2 Cannabinoid Receptors Promote Neural Progenitor Cell Proliferation via mTORC1 Signaling * Journal of Biological Chemistry 287, 1198-1209.

Palazzo, I., Deistler, K., Hoang, T.V., Blackshaw, S., and Fischer, A.J. (2019). NF-kB signaling regulates the formation of proliferating Müller glia-derived progenitor cells in the avian retina. BioRxiv 724260.

Palazzo, I., Deistler, K., Hoang, T.V., Blackshaw, S., and Fischer, A.J. (2020). NF-kB signaling regulates the formation of proliferating Müller glia-derived progenitor cells in the avian retina. Development.

Pertwee, R.G. (2010). Receptors and Channels Targeted by Synthetic Cannabinoid Receptor Agonists and Antagonists. Curr Med Chem 17, 1360-1381.

Rapino, C., Tortolani, D., Scipioni, L., and Maccarrone, M. (2018). Neuroprotection by (Endo)Cannabinoids in Glaucoma and Retinal Neurodegenerative Diseases. Curr Neuropharmacol 16, 959-970.

Satija, R., Farrell, J.A., Gennert, D., Schier, A.F., and Regev, A. (2015). Spatial reconstruction of single-cell gene expression data. Nat Biotechnol 33, 495-502.

Shamsuddin, N., and Kumar, A. (2011). TLR2 mediates the innate response of retinal Muller glia to Staphylococcus aureus. J Immunol 186, 7089-7097.

da Silva Sampaio, L., Kubrusly, R.C.C., Colli, Y.P., Trindade, P.P., Ribeiro-Resende, V.T., Einicker-Lamas, M., Paes-de-Carvalho, R., Gardino, P.F., de Mello, F.G., and De Melo Reis, R.A. (2018). Cannabinoid Receptor Type 1 Expression in the Developing Avian Retina: Morphological and Functional Correlation With the Dopaminergic System. Front Cell Neurosci 12.

Silverman, S.M., and Wong, W.T. (2018). Microglia in the Retina: Roles in Development, Maturity, and Disease. Annual Review of Vision Science 4, 45-77.

727 Slusar, J.E., Cairns, E.A., Szczesniak, A.-M., Bradshaw, H.B., Di Polo, A., and Kelly, M.E.M. neuroprotection in a rat model of optic nerve axotomy. Neuropharmacology 72, 116-125. expression in glial cells in the retina and optic nerve of birds and mammals. J Comp Neurol 518, 2316-2333. 253.

Stincic, T.L., and Hyson, R.L. (2011). The localization and physiological effects of cannabinoid receptor 1 (CB1) in the brain stem auditory system of the chick. Neuroscience 194C, 150-159. 
Straiker, A.J., Maguire, G., Mackie, K., and Lindsey, J. (1999). Localization of Cannabinoid CB1 Receptors in the Human Anterior Eye and Retina. Invest. Ophthalmol. Vis. Sci. 40, 2442-2448.

739 Todd, L., and Fischer, A.J. (2015). Hedgehog-signaling stimulates the formation of proliferating Müller glia-derived progenitor cells in the retina. Development 142, 2610-2622.

741 Todd, L., Palazzo, I., Suarez, L., Liu, X., Volkov, L., Hoang, T.V., Campbell, W.A., Blackshaw,

742 S., Quan, N., and Fischer, A.J. (2019). Reactive microglia and IL1ß/IL-1R1-signaling mediate

743 neuroprotection in excitotoxin-damaged mouse retina. Journal of Neuroinflammation 16, 118.

744 Todd, L., Finkbeiner, C., Wong, C.K., Hooper, M.J., and Reh, T.A. (2020). Microglia Suppress

745 Ascl1-Induced Retinal Regeneration in Mice. Cell Reports 33, 108507.

746 Ullrich, O., Merker, K., Timm, J., and Tauber, S. (2007). Immune control by endocannabinoids

747 - New mechanisms of neuroprotection? Journal of Neuroimmunology 184, 127-135.

748 White, D.T., Sengupta, S., Saxena, M.T., Xu, Q., Hanes, J., Ding, D., Ji, H., and Mumm, J.S. (2017). Immunomodulation-accelerated neuronal regeneration following selective rod

750 photoreceptor cell ablation in the zebrafish retina. PNAS 114, E3719-E3728.

751 Xu, J.-Y., and Chen, C. (2015). Endocannabinoids in Synaptic Plasticity and Neuroprotection.

752 Neuroscientist 21, 152-168.

753 Yang, W., Li, Q., Wang, S.-Y., Gao, F., Qian, W.-J., Li, F., Ji, M., Sun, X.-H., Miao, Y., and

Wang, Z. (2016). Cannabinoid receptor agonists modulate calcium channels in rat retinal müller cells. Neuroscience 313, 213-224.

Yazulla, S., Studholme, K.M., McINTOSH, H.H., and Fan, S.-F. (2000). Cannabinoid receptors on goldfish retinal bipolar cells: Electron-microscope immunocytochemistry and whole-cell

758 recordings. Visual Neuroscience 17, 391-401.

Zelinka, C.P., Volkov, L., Goodman, Z.A., Todd, L., Palazzo, I., Bishop, W.A., and Fischer, A.J. (2016). mTor signaling is required for the formation of proliferating Müller glia-derived progenitor

761 cells in the chick retina. Development 143, 1859-1873. 
768 Figure legends:

Figure 1. eCB-related genes are widely expressed in different types of retinal

cells. Panel a illustrates a schematic diagram of the enzymes and receptors involved

773 of expression of eCB-related genes among retinal cells. Patterns and levels of

774 expression are presented in UMAP plots (b-h) and violin plots (i). scRNA-seq libraries

775 were aggregated from control and treated $3 \mathrm{hr}, 12 \mathrm{hr}$, and 48hr after NMDA-treatment

776 (b). UMAP-ordered cells formed distinct clusters of neuronal cells, resting MG, early

777 activated MG, activated MG and late activated MG (c-e). UMAP heatmaps of CNR1,

778 MGLL, NAPEPLD, DAGLA, DAGLB and FAAH demonstrate patterns and levels of

779 expression across different retinal cells, with black dots representing cells with

780 expression of 2 or more genes (f-h). Violin plots illustrate relative levels and percent of

781 expression in resting and activated MG (i). Violin plots illustrate levels of gene

782 expression and significant changes $\left({ }^{*} p<0.01,{ }^{* *} p<10 \exp -10,{ }^{* *} p<10 \exp -20\right)$ in levels

783 that were determined by using a Wilcox rank sum with Bonferroni correction.

785 Figure 2. eCB-related genes are dynamically expressed by MG in response to

786 damage or growth-factor treatment. scRNA-seq was used to identify patterns of

787 expression of eCB genes in MG at several time points after NMDA damage or FGF +

788 insulin growth factor treatment to form MGPCs. UMAP- clusters of MG were identified

789 by expression of hallmark genes $(\mathbf{a}, \mathbf{b}, \mathbf{d})$. Progenitors were then classified by different

790 cell cycle and progenitor markers $(\mathbf{c}, \mathbf{e}, \mathbf{f})$. Each dot represents one cell and black dots 
791 indicate cells with 2 or more genes expressed. The expression of eCB related genes

792 was illustrated in a colored heatmap and in a violin plot violin plot with population

793 percentages and statistical comparisons. $(\mathbf{g}, \mathbf{h}, \mathbf{i})$. Significant difference $\left({ }^{*} \mathrm{p}<0.01\right.$,

$\left.794{ }^{* *} \mathrm{p}<0.0001,{ }^{* *} \mathrm{p}<<0.0001\right)$ was determined by using a Wilcox rank sum with Bonferroni

795 correction. MG - Müller glia, MGPC - Müller glia-derived progenitor cell.

Figure 3. eCB increase numbers of proliferating MGPCs in damaged retinas.

798 Chick eyes were injected with NMDA, AEA, 2-AG, and EdU according to the paradigm

799 at the top figure. Eyes were harvested at $24 \mathrm{hrs}$ after the last injection and retinas

800 processed for immunolabeling. Retinas were labeled for Sox2 (green) and EdU (red)

801 cells (a), neurofilament (red), phospho-Histone H3 (pHisH3, green), and DAPI (blue; d).

802 Dying cells were labeled using the TUNEL assay (e). Histograms illustrate the mean $( \pm$ 803 SD) and each dot represents one biological replicate. Significance of difference ( $\left.804{ }^{* *} \mathrm{p}<0.01,{ }^{* * *} \mathrm{p}<0.001\right)$ was determined by using a paired $t$-test. Arrows indicate the 805 nuclei of MG. The calibration bar panels a, $\mathbf{d}$ and e represent $50 \mu \mathrm{m}$. Abbreviations:

806 ONL - outer nuclear layer, INL - inner nuclear layer, IPL - inner plexiform layer, GCL 807 ganglion cell layer, NF - neurofilament, ns - not significant.

809 Figure 4. Small molecule drug targeting CNR1, DAGL and MGLL influence the

810 formation of MGPCs. The treatment paradigm is illustrated at the top of the figure.

811 Compounds included Orlistat (DAGL inhibitor), JJKK048 (MGLL inhibitor), Win55

812 (CNR1 agonist), and Rimonabant (CNR1 antagonist). Competitive inhibitor ELISAs of

813 illustrate relative levels of 2-AG and AEA after NMDA damage and treatment with

814 Orlistat or JJKK048 (a,b). Retinas were labeled for Sox2 (green) and EdU (red; a). 
815 Arrows indicate EdU+/Sox2+ nuclei of MGPCs, small double-arrows indicate

816 EdU+/Sox2+ nuclei of NIRG cells in the IPL, and hollow arrow-heads indicate

817 EdU+/Sox- nuclei of presumptive microglia. The histograms in $\mathbf{a}, \mathbf{b}$ and $\mathbf{d}-\mathbf{g}$ represents

818 the mean $( \pm S D)$ and each dot represents one biological replicate retina. The calibration

819 bar in c represents $50 \mu \mathrm{m}$. Abbreviations: ONL - outer nuclear layer, INL - inner

820 nuclear layer, IPL - inner plexiform layer, GCL - ganglion cell layer.

821

822

Figure 5. Targeting the AEA pathway does not influence the formation of MGPCs.

823 The treatment paradigm is illustrated at the top of the figure. Compounds included

824 ARN19874 (NAPEPLD inhibitor), URB597 (FAAH inhibitor) and PF-044 (FAAH

825 inhibitor). Eyes were harvested at $24 \mathrm{hrs}$ after the last injection and retinas processed

826 for immunofluorescence. Retinal sections were labeled for Sox2 (green) and EdU (red;

827 a), CD45 (green) and EdU (red; c), Nkx2.2 (green) and EdU (red, e), and cell death

828 (TUNEL, red; $\mathbf{g}$ ). The histograms in $\mathbf{b}, \mathbf{d}, \mathbf{f}, \mathbf{h}$ represents the mean $( \pm \mathrm{SD})$ number of

829 proliferating MGPCs (b), proliferating microglia (d), proliferating NIRG cells (f), and

830 dying cells (h). Each dot represents one biological replicate retina. Arrows indicate

831 EdU+/Sox2+ nuclei of MGPCs, small double-arrows indicate EdU+/Nkx2.2+ nuclei of

832 NIRG cells in the IPL, and hollow arrow-heads indicate EdU+/CD45+ nuclei of microglia.

833 The calibration bar in $\mathbf{g}$ represents $50 \mu \mathrm{m}$ and applies to panels $\mathbf{a , c , e}$ and $\mathbf{g}$.

834 Abbreviations: ONL - outer nuclear layer, INL - inner nuclear layer, IPL - inner

835 plexiform layer, GCL - ganglion cell layer. Microglia were from aggregate scRNA-seq

836 libraries were re-embedded and ordered in UMAP (i). Microglia from saline- and NMDA-

837 treated retinas were clustered into resting, active and early activated cells (j). Clusters 
838 of cells were arranged according to markers such as TNFSF15, PPARG, IL1R2, DBI

839 and $A I R 1 L(\mathbf{k}, \mathbf{l})$. MGLL and NAPEPLD had scattered expression across microglia in

840 different clusters, whereas CNR1 was not widely expressed (m).

842 Figure 6. Microglia reactivity in damaged retina treated with eCBs. The treatment

843 paradigm is illustrated at the top of the figure. Compounds included 2-AG+AEA (CNR1

844 agonists), Orlistat (DAGL inhibitor), JJKK048 (MGLL inhibitor), Win55 (CNR1 agonist), 845 and Rimonabant (CNR1 antagonist). Eyes were harvested 24hrs after the last injection 846 and retinas were processed for immunofluorescence. Retinal sections were labeled for

847 CD45 (green) and DAPI (blue; a), or CD45 (green), EdU (red) and DAPI (blue; b).

848 Microglial reactivity was assessed by measuring CD45 area and intensity, proliferation

849 (EdU+), and total number of microglia (CD45+/DRAQ5+) (b). Arrows indicate the nuclei

850 of microglia. Histograms in c illustrate the mean $( \pm S D$, control $n=40$, treatment $n=8)$

851 and each symbol represents one biological replicate. The shape of the microglia was

852 assessed using a Sholl analysis. Representative microglia from each condition is

853 shown, with a heat map of radial intersections (blue $=$ low, red/white $=$ high) $(\mathbf{c})$. The

854 graph in d illustrates of the number of processes radially $(\mu \mathrm{m} \pm S E)$ from microglial nuclei

855 from control $(n=15)$ and NMDA damaged $(n=25)$ retinas. (d) Significance of difference

856 was determined by using a one way ANOVA with corresponding Tukey's test. The

857 calibration bars panels a, b represent $50 \mu \mathrm{m}$, and the bar in d represents $5 \mu \mathrm{M}$.

858 Abbreviations: ONL - outer nuclear layer, INL - inner nuclear layer, IPL - inner

859 plexiform layer, GCL - ganglion cell layer. 
Figure 7. Patterns of expression of eCB-related genes in normal and NMDA-

damaged mouse retinas. Cells were obtained from control retinas and from retinas at

864 each dot representing an individual cell (a). UMAP plots revealed distinct clustering of different types of retinal cells; resting MG (a mix of control, 48hr and 72hr NMDA-tr), 12$72 \mathrm{hr}$ NMDA-tr MG (activated MG in violin plots), 6hrs NMDA-tr MG, 3hrs NMDA-tr MG,

867 microglia, astrocytes, RPE cells, endothelial cells, retinal ganglion cells, horizontal cells

868 (HCs), amacrine cells (ACs), bipolar cells (BPs), rod photoreceptors, and cone

869 photoreceptors (b). Resting and activated MG were identified based on patterns of

870 expression of Slc1a3, Nes and $\operatorname{Vim}(\mathbf{c})$. Cells were colored with a heatmap of

871 expression of Cnr1, Daglb, Mgll, Napepld and Faah expression (d-f). Black dots indicate

872 cells that express two or more markers.

Figure 8. eCBs and NF-kB-signaling in MG of damaged mouse retinas

875 The treatment paradigm is illustrated at the top of the figure. Eyes of mice (cis-NF-

$876 \mathrm{kB}^{\text {eGFP}}$ ) were pretreated with compounds or vehicle prior to NMDA + vehicle/compound,

877 and retinas harvested 24 hrs after the last injection. Compounds included 2-AG+AEA

878 (CNR1 agonists), Win55 (CNR1 agonist), and Rimonabant (CNR1 antagonist). Retinal sections were labeled for Sox9 (blue) and eGFP (green) (b), fragmented DNA using the

880 TUNEL method (d), and Iba1 (red) and Draq5 (blue; f). The histogram/scatter-plots

881 illustrate the mean $( \pm S D)$ number of eGFP+MG (c), dying cells (e) or lba1+/Draq5+

882 cells $(\mathbf{g})$. Each dot represents one biological replicate. Significance of difference

$883\left({ }^{*} \mathrm{p}<0.05\right)$ was determined by using a paired $t$-test. The calibration bars panels $\mathbf{a}, \mathbf{c}, \mathbf{e}$, 
884 and $\mathbf{g}$ represent $50 \mu \mathrm{m}$. Abbreviations: ONL - outer nuclear layer, INL - inner nuclear

885 layer, IPL - inner plexiform layer, $\mathrm{GCL}$ - ganglion cell layer.

886

887 Figure 9. Summary Schematic of eCB effect on MG reprogramming. Using scRNA-

888 seq analysis, we identified patterns of expression of genes involved in eCB synthesis,

889 degradation and signaling (a). ELISAs indicated a more prominent role of 2-AG over

890 AEA, and drugs which elevated 2-AG signaling increased MGPCs after damage (b).

891 Microglia were unresponsive to these treatments and retained a reactive phenotype in a

892 damaged retina (c). In the NF-kBeGFP+ reporter mice, damage activates signaling in

893 MG, which is decreased by exogenous eCBs or CNR1 agonists (d).

894

895 Table 1. Sholl Analysis of microglia from the chick retina after damage and eCB

896 treatment. Statistical analysis was performed with a one-way ANOVA and Tukey's test

$897($ NMDA $n=25$, treatments $n=15)$ 


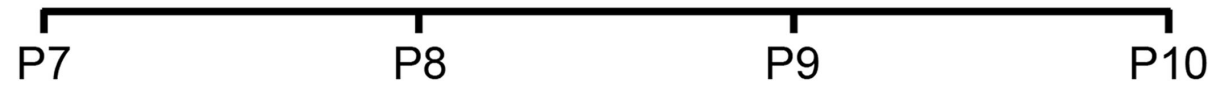
control NMDA + vehicle
$\mathrm{EdU}+$ vehicle
$\mathrm{EdU}+$ vehicle
harvest
treated NMDA + compound
EdU + compound
EdU + compound
harvest
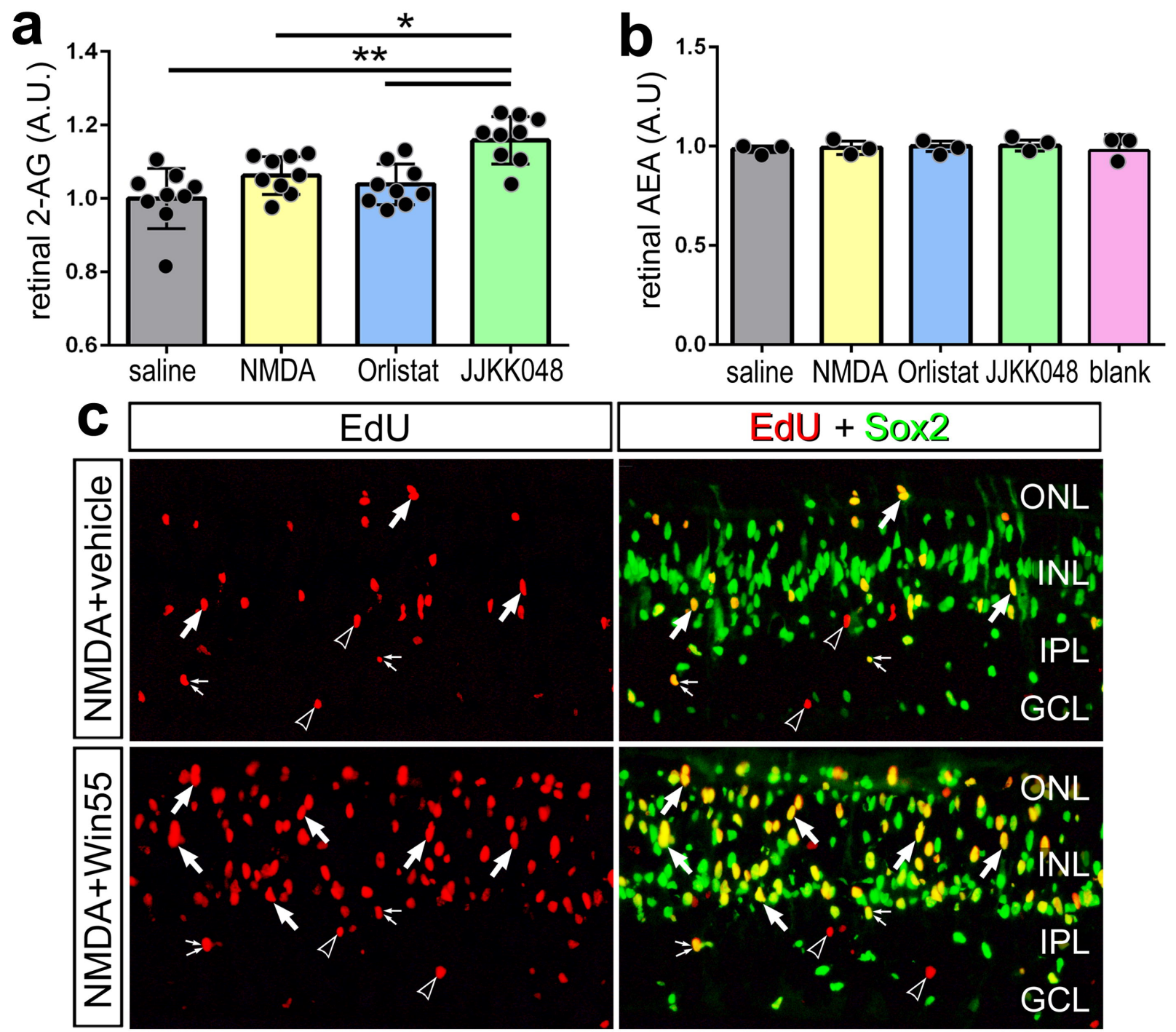

d

个CNR1 activity MGPCs proliferation

0
$\overline{0}$
+
$\overline{\mathbf{z}}$

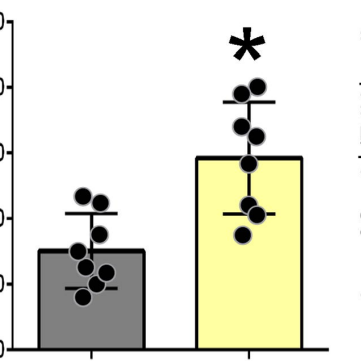

NMDA NMDA+Win55
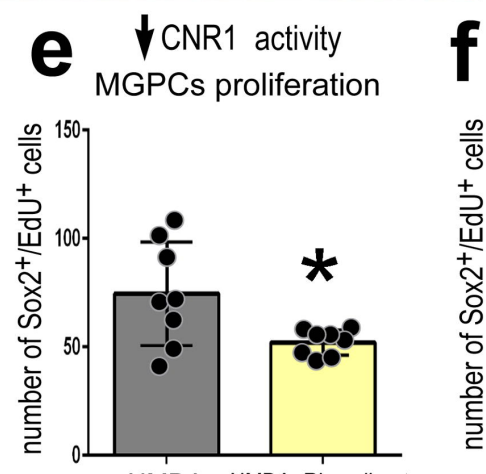

NMDA NMDA+Rimonibant

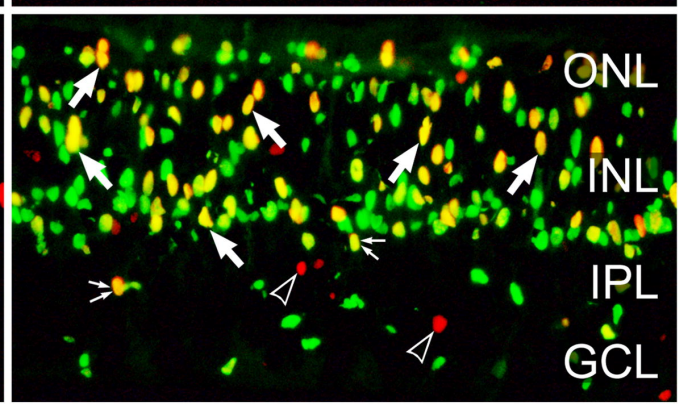

†2-AG Q MGPCs proliferation
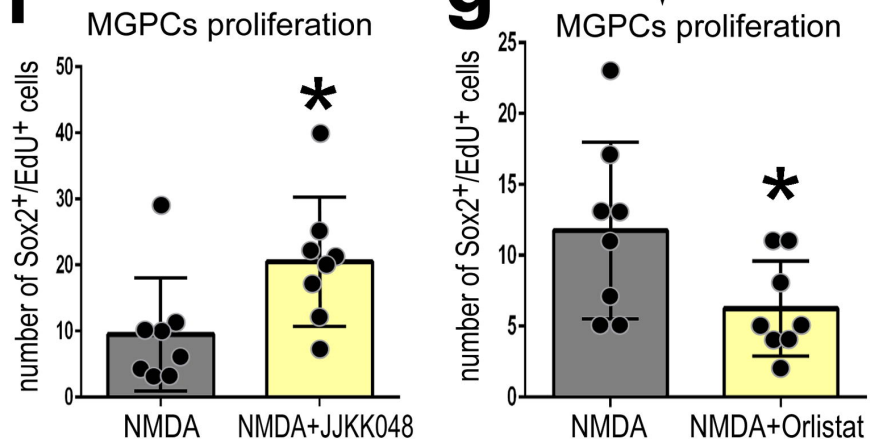

NMDA NMDA+Orlistat 


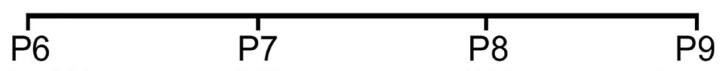

control NMDA+vehicle treated NMDA+ARN19874 or (NAPEPLD inhibitor) treated NMDA+URB597 or PF-044 EdU+

(FAAH inhibitors) URB597 or PF-044 URB597 or PF-044

P9 harvest

harvest

proliferating MGPCs

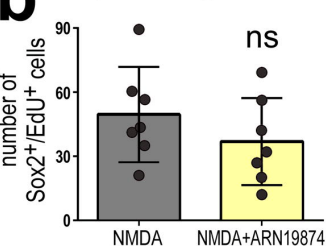

d
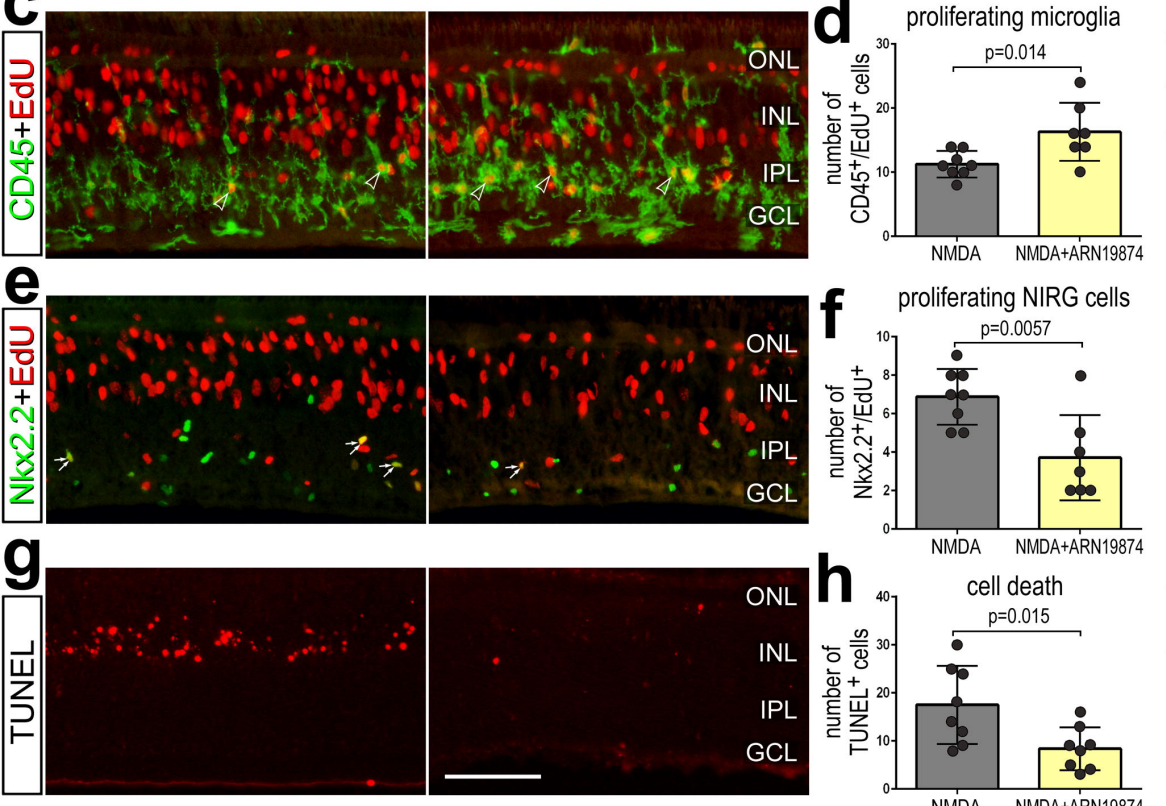
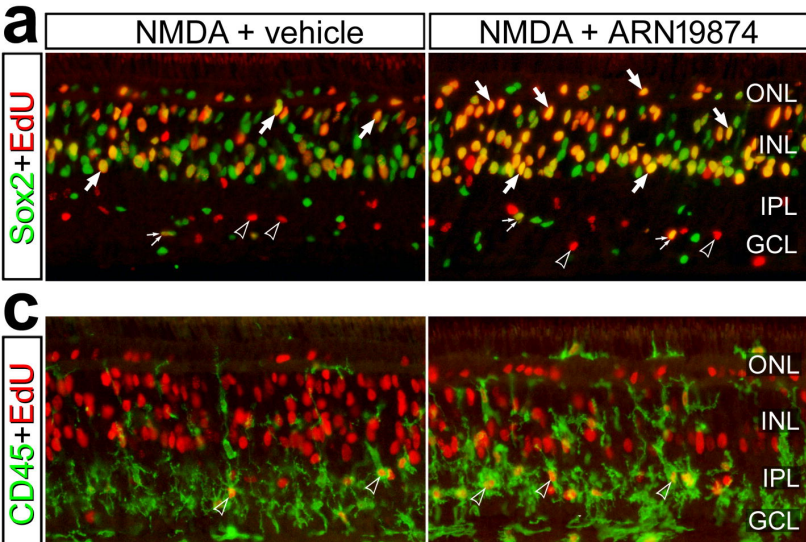

a

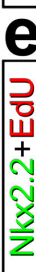

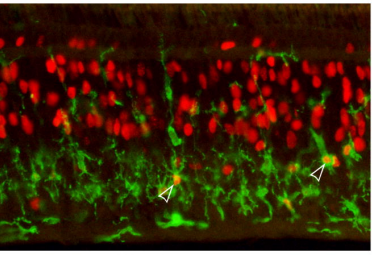
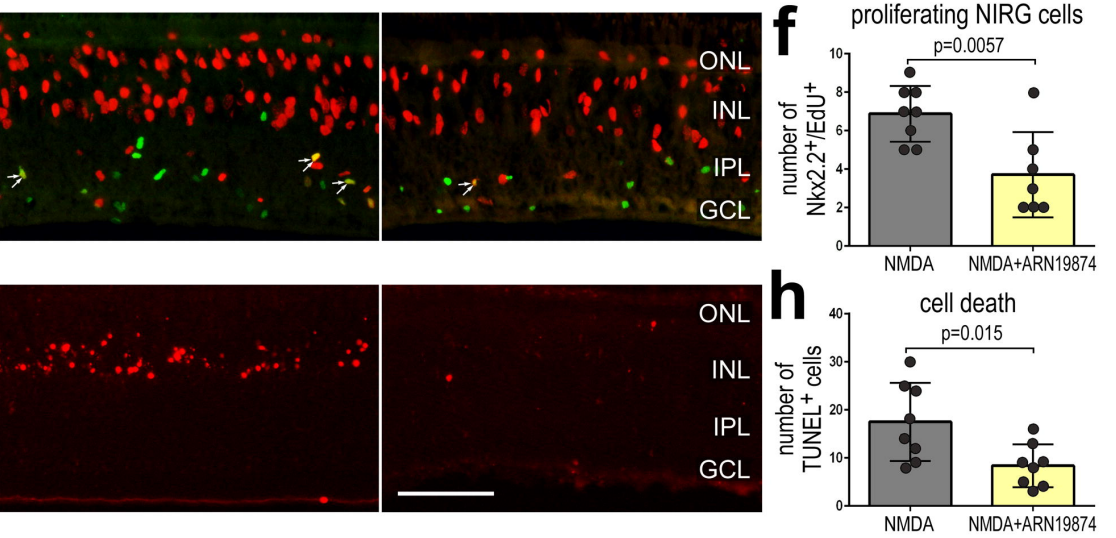

ARN19874

proliferating MGPCs
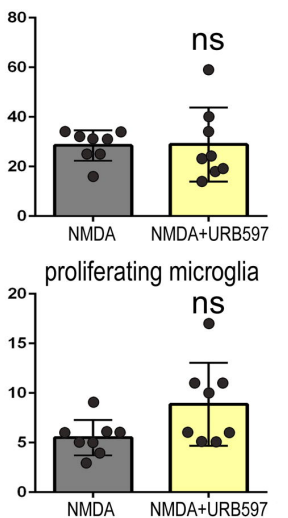

proliferating NIRG cells
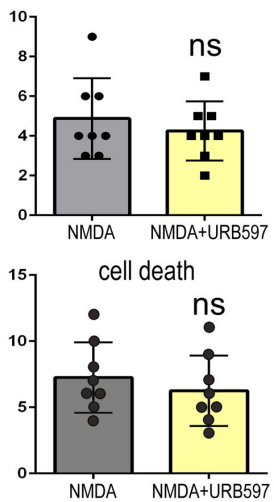

PF-044

proliferating MGPCs

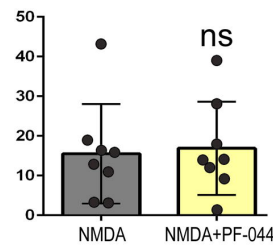

proliferating microglia

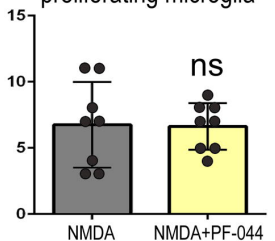

proliferating NIRG cells
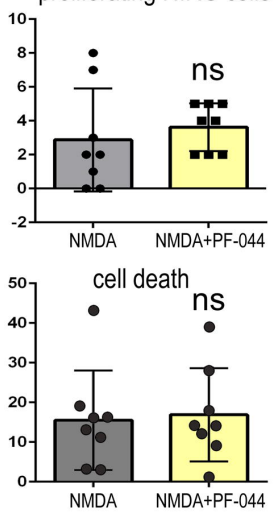
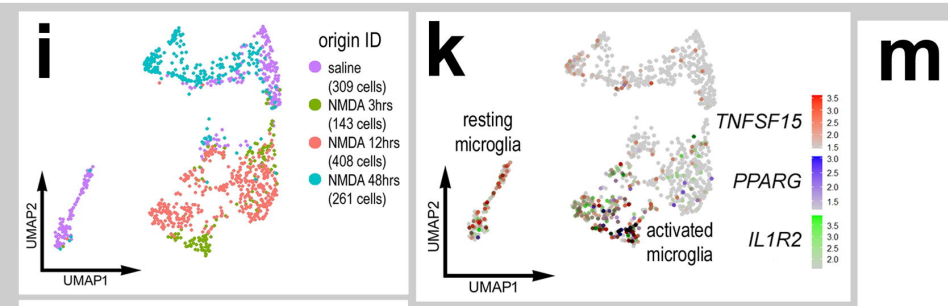

(
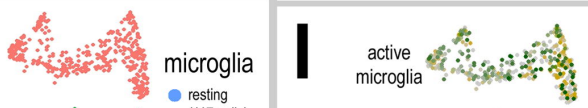

ôt

- resting

(117 cells)
active
$(415$ cells)

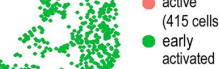

activated
$(589$ cells $)$
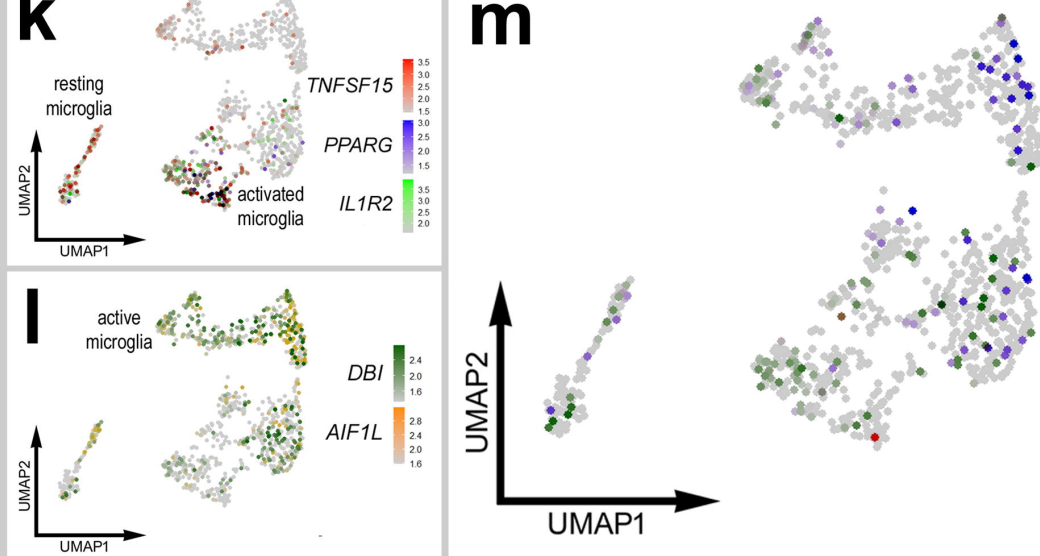

CNR1

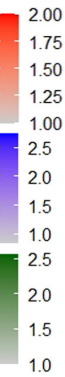

UMAP1 


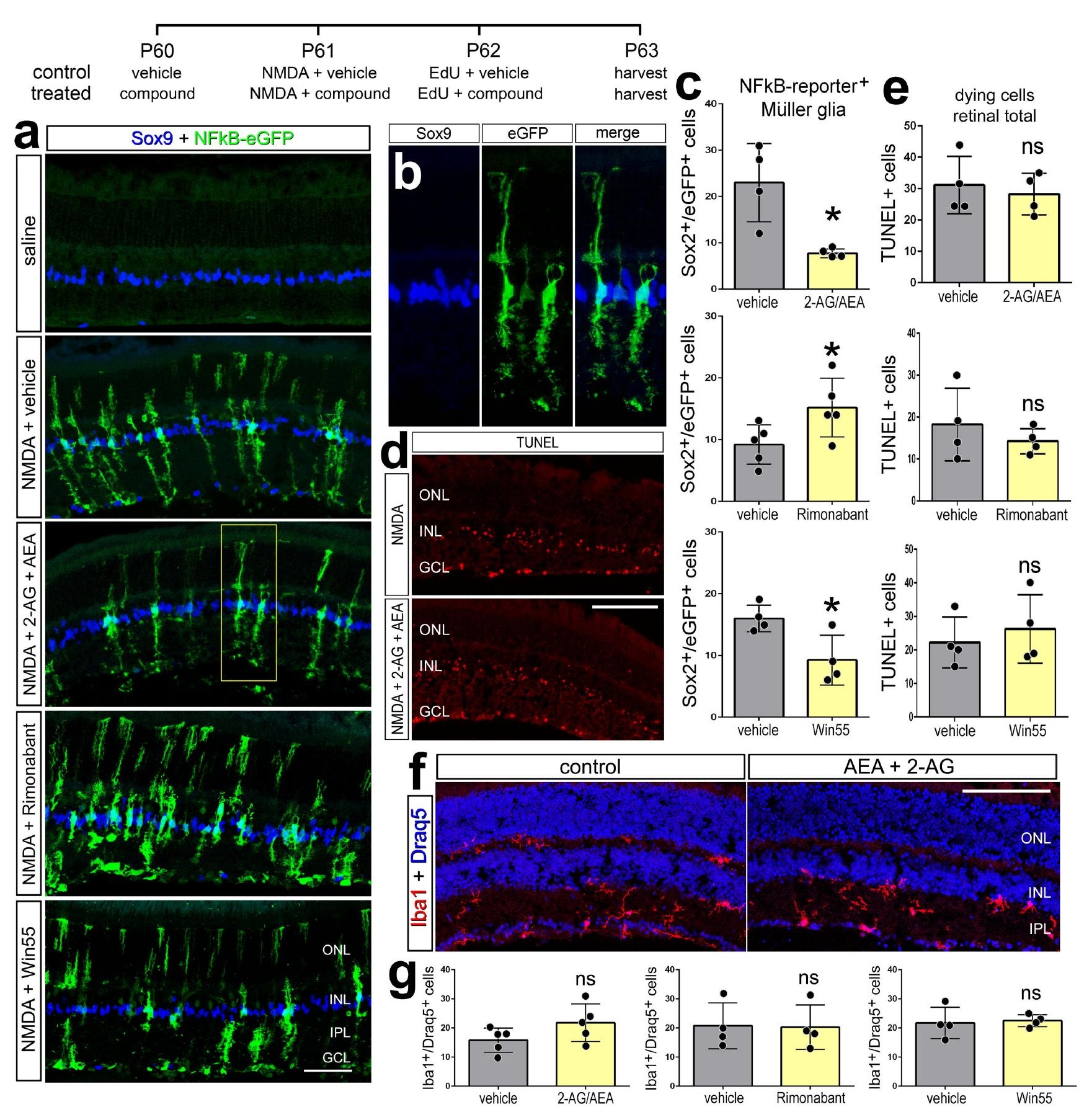


Microglia Morphology

max inters. radius $(\mu \mathrm{M})$

mean intersections

$12.26 \pm 4.01 \quad 11.90 \pm 3.72$

$8.40 \pm 1.75$

$3.46 \pm 0.85$

\section{enhance signal}

dampen signal

JJKK-048

Win55-212,2

Rimonabant

$11.99 \pm 3.51$

$8.80 \pm 2.54$

$12.65 \pm 5.44$

$7.40 \pm 1.76$

$14.01 \pm 5.83$

$8.20 \pm 1.85$

$12.27 \pm 2.96$

$9.13 \pm 1.88$

$0.36 \pm 0.68$

ANOVA

$3.36 \pm 0.61$ 